\title{
Current status of treatment of metastatic colorectal cancer with special reference to cetuximab and elderly patients
}

\author{
Per Pfeiffer \\ Camilla Qvortrup \\ Jon K Bjerregaard
}

Department of Oncology, Odense

University Hospital. Institute of

Clinical Research, University of

Southern Denmark. Odense C,

Denmark
Correspondence: Per Pfeiffer Department of Oncology, Odense University Hospital, Sdr Boulevard 29, DK - 5000 Odense C, Denmark Email per.pfeiffer@ouh.regionsyddanmark.dk
Purpose: Elderly cancer patients often have co-morbidities and other characteristics that make the selection of optimal treatment more complex. The introduction of targeted therapies in colorectal cancer has further complicated this problem. This review will focus on the role of the EGFR antibody cetuximab in elderly patients.

Methods: We have reviewed the available evidence in the literature to evaluate the results of therapy with cetuximab, alone or in combination with chemotherapy, with a focus on elderly patients with metastatic colorectal cancer (mCRC).

Results: In patients with mCRC, combination chemotherapy prolongs median survival to more than 18 months and even around 24 months in combination with cetuximab in selected patients. No prospective studies have evaluated cetuximab in elderly patients. However, subgroup analyses from randomized trials and retrospective analysis suggest that the efficacy of chemotherapy and cetuximab is maintained in fit elderly patients, but with slightly increased but acceptable toxicity.

Conclusion: No prospective cetuximab studies have been conducted solely in a population of elderly patients. However, available data suggest that outcomes in the fit elderly mirror results observed in younger patients.

Keywords: metastatic colorectal cancer, cetuximab, elderly patients

\section{Introduction}

WHO defines an elderly person in the developed countries as a person with a chronological age of 65 years and above (65+ years). However aging is a heterogeneous process and the 'chronological age' is not always predicting of the 'physiological age'. In many but not all clinical studies, 65+ years is used as cut-point for evaluating efficacy and toxicity in younger and older patients.

The median age of patients with colorectal cancer (CRC) is 72 years at the time of the primary diagnosis. Approximately $70 \%$ of CRC patients are aged $65+$ years and $40 \%$ are aged $75+$ years thus making CRC cancer a disease primarily of the elderly. Despite this fact the elderly are under-represented in clinical trials. ${ }^{1-3}$ In addition, co-morbidity is often an exclusion criterion in clinical trials and thus elderly patients in clinical trials constitute a highly selected group. Therefore it is often difficult to extrapolate results from clinical trials to the daily practice of treating the elderly patient.

In less than a decade the research and development of medical treatment modalities in patients with metastatic colorectal cancer (mCRC) have changed the treatment options from monotherapy with 5-fluorouracil (FU) modulated by folinic acid (FA) to combination chemotherapy with irinotecan or oxaliplatin and very recently to chemotherapy in combination with targeted therapy. ${ }^{4}$

In 2004 two targeted therapies were approved in the USA and the EU for patients with $\mathrm{mCRC}$, and are now used in daily practice: Cetuximab (Erbitux $\left.{ }^{\circledR}\right)$, a monoclonal 
antibody blocking the epidermal growth factor receptor (EGFR) and bevacizumab (Avastin ${ }^{\circledR}$ ) a monoclonal antibody targeting angiogenesis. Since then, panitumumab (Vectibix ${ }^{\circledR}$ ), a human antibody against EGFR, has been approved as monotherapy in patients with KRAS wild-type mCRC. 5,6

In this review we will summarize the present status of cetuximab in patients with $\mathrm{mCRC}$ with special attention to elderly patients. As cetuximab, is most frequently used in combination with chemotherapy, we will briefly summarize the current principles of chemotherapy in patients with mCRC.

\section{Methods}

To identify data on therapy with cetuximab in elderly patients with mCRC, we searched the databases Medline and ClinicalTrials.gov for relevant publications using the search terms colon cancer, CRC, elderly antibody therapy, monoclonal antibody, targeted therapy, cetuximab. Data on treatment and side effects were also identified in relevant publications and from listings in recent overviews. Full reporting of final results from important clinical trials often lags behind more preliminary reports in the abstract form. Therefore, we included data from abstracts to be able to present the most recent information on treatment. Abstracts presented at the annual meetings of ASCO, ASCO GI, AACR, ECCO/ ESMO from 2005 to 2008 were reviewed and included as applicable.

\section{Palliative chemotherapy in patients with metastatic CRC}

The modern era of combination chemotherapy started when it was shown that irinotecan prolonged median overall survival (OS) in patients resistant to FU/FA. ${ }^{7,8}$ Since then, the use of combination chemotherapy, both as first and second line, has increased the life expectancy to nearly 2 years. ${ }^{9,10}$

First-line doublets (Table 1) increase response rates from $20 \%$ to more than $40 \%$ and prolong progression-free survival (PFS) from 4 to 6 months to 6 to 8 months. ${ }^{9}$ Direct comparisons between different combinations (Table 1) with irinotecan (eg, FOLFIRI or FLIRI) and oxaliplatin (eg, FOLFOX, XELOX or FLOX) demonstrate that nearly all doublets are equally effective with respect to response rate, median PFS and median OS. ${ }^{1-13}$ The most important exception to this statement is IFL which is too toxic and less active and should no longer be offered as a standard regimen. ${ }^{14}$

OS is correlated with the percentage of patients who receive all three agents (FU/FA, irinotecan and oxaliplatin) and the use of first- and second-line combination chemotherapy

Table I Selected randomized studies evaluating chemotherapy in patients with metastatic colorectal cancer

\begin{tabular}{|c|c|c|c|c|c|}
\hline Author, year & Regimen & No of patients & RR (\%) & Median PFS (months) & Median OS (months) \\
\hline \multicolumn{6}{|c|}{ FU/FA versus combination with irinotecan } \\
\hline \multirow[t]{2}{*}{ Saltz et al $2000^{96}$} & FU/FA & 226 & 21 & 4.3 & 12.6 \\
\hline & IFL & 231 & $39 *$ & $7.0^{*}$ & $14.8^{*}$ \\
\hline \multirow[t]{2}{*}{ Douillard et al $2000^{97}$} & FU/FA & 187 & 22 & 4.4 & 14.1 \\
\hline & FOLFIRI & 198 & $35^{*}$ & $6.7^{*}$ & $17.4^{*}$ \\
\hline \multirow[t]{2}{*}{ Köhne et al $2005^{98}$} & FU/FA & 216 & 32 & 6.4 & 16.9 \\
\hline & FUFIRI & 214 & $54 *$ & $8.5^{*}$ & 20.1 \\
\hline \multicolumn{6}{|c|}{ 5-FU/FA versus combination with oxaliplatin } \\
\hline \multirow[t]{2}{*}{ de Gramont et al $2000^{99}$} & FU/FA & 210 & 22 & 6.2 & 14.7 \\
\hline & FOLFOX & 210 & $51 *$ & $9.0^{*}$ & 16.2 \\
\hline \multirow[t]{2}{*}{ Giacchetti et al $2000^{100}$} & FU/FA & 100 & 12 & 6.1 & 19.9 \\
\hline & FOLFOX & 100 & $34 *$ & $8.7^{*}$ & 19.4 \\
\hline \multicolumn{6}{|c|}{ Combination versus combination } \\
\hline \multirow[t]{2}{*}{ Tournigand et al 2004"I } & FOLFOX & 111 & 54 & 10.9 & 20.6 \\
\hline & FOLFIRI & 111 & 56 & 14.2 & 21.5 \\
\hline \multirow[t]{2}{*}{ Goldberg et al $2004^{14}$} & IFL & 264 & 31 & 6.9 & 15.0 \\
\hline & FOLFOX & 267 & $45^{*}$ & $8.7^{*}$ & $19.5^{*}$ \\
\hline \multirow[t]{2}{*}{ Glimelius et al $2008^{12}$} & FLIRI & 281 & 35 & 9.4 & 19.4 \\
\hline & FOLFIRI & 286 & $49 *$ & 9.0 & 19.0 \\
\hline \multirow[t]{2}{*}{ Cassidy et al $2008^{13}$} & XELOX & 1017 & 47 & 8.0 & 19.8 \\
\hline & FOLFOX & 1017 & 48 & 8.5 & 19.6 \\
\hline
\end{tabular}

*Significant difference.

Abbreviations: FU/FA, 5-fluorouracil/folinic acid; RR, response rate; PFS, progression-free survival; OS, overall survival. 
will assure that more patients are exposed to the three keys drugs. ${ }^{10,15-17}$ It is considered of minor importance which therapy is used as first-line treatment and the general opinion at present is that the choice of first-line therapy is mostly a matter of the adverse effects profile..$^{10,15-17}$

The most important and the most dangerous side effect from irinotecan is diarrhea, and careful information is very important.

The dose-limiting side effect of continuous therapy with oxaliplatin is chronic sensory neuropathy limiting the use of oxaliplatin beyond a cumulative dose of $1000 \mathrm{mg} / \mathrm{m}^{2}$, corresponding to 6 months of therapy.

Many patients are still in an excellent performance status despite progressive disease after treatments with different combinations of FU/FA, irinotecan and oxaliplatin. Third-line chemotherapy in these patients has poor efficacy with response rate less than 5\%, PFS less than 2 months and OS around 4 months and is not recommended outside clinical trials. ${ }^{18}$ The availability of targeted therapy has opened up new possibilities for these patients.

\section{Duration of combination chemotherapy}

At the time when FU/FA was the only choice, treatment was maintained until progression. This strategy was carried on with the introduction of doublets. However this strategy has to be revised as recent studies have shown that an intermittent strategy compared with continuous use of chemotherapy does not compromise efficacy. ${ }^{19,20}$

The overall conclusion of OPTIMOX1 and OPTIMOX2 was that a total pause in treatment can not be recommended after only 3 months of treatment due to an inferior OS. ${ }^{19,21}$ However single-drug treatment or biological treatment may be used as maintenance therapy. ${ }^{19,21}$ A new analysis of these data showed that a treatment pause is fully acceptable if therapy is given for at least 6 months. ${ }^{22}$

\section{Elderly patients tolerate and benefit from combination chemotherapy}

Several studies have shown that elderly patients enrolled in trials have similar benefits and comparable toxicity profiles of single agent fluoropyrimidines compared to younger patients. $^{23-26}$

The only exception is neutropenia, which was found more often in elderly patients in a pooled analysis of 7 adjuvant trials with single agent FU. ${ }^{27}$

In a pooled analysis of safety and efficacy of FOLFOX4 in 3742 patients included in 4 clinical trials (adjuvant and palliative therapy) it was shown that FOLFOX4 maintains its efficacy and safety ratio in elderly patients (70+ years). However, grade 3-4 neutropenia and thrombocytopenia were observed more frequently in elderly patients. ${ }^{28}$

In a recent combined analysis of more than 2500 patients treated with different irinotecan/FU schedules in 4 first-line phase III trials, the authors concluded that elderly patients (70+ years) who fulfilled the inclusion criteria of these trials had similar benefits of treatment and similar risk of toxicity as younger patients and these results were also confirmed in systematic reviews. ${ }^{29-31}$

Nevertheless, a recent large community-based study demonstrated that elderly patients (age 65+ years) were less likely to receive first-line doublet chemotherapy and also less likely to receive irinotecan, oxaliplatin, and bevacizumab during the entire course of the disease. In this unselected group of patients the elderly had a shorter median survival (19.1 months versus 24.5 months) and more toxicity-related hospitalizations (21\% versus $11 \%)$ than the younger patients. ${ }^{32}$ This discrepancy between the results from subgroup analyses in randomized clinical trials and this community-based study is probably due to a higher proportion of patients with co-morbidities and poorer performance status in the unselected community-based study than reported in randomized clinical trials. Therefore it is important to distinguish between the frail elderly patients with co-morbidity and poor performance status and the fit elderly patient, as fit elderly tolerate combination chemotherapy and have the same benefit as younger patients. ${ }^{30,31}$

\section{Cetuximab for treatment of metastatic CRC}

\section{Pharmacodynamics}

Cetuximab is an human-murine chimeric antibody directed against the ligand-binding site of EGFR. The EGFR is a transmembrane glycoprotein that is involved in signaling pathways affecting cellular growth, differentiation, and proliferation. ${ }^{33}$ It is a member of the HER tyrosine kinase growth factor receptor family and is expressed in many types of normal tissues. Binding of ligands to the extracellular domain promotes dimerization of the HER receptor family, and activates intracellular downstream kinases. Downstream effectors are among others the RAF/MEK/ERK pathway as well as the PI3K/PTEN/AKT pathway. Central to both of these pathways are RAS regulatory proteins, among these KRAS.

Up-regulation of the EGFR is found in a large number of cancers. Sixty to eighty percent of CRC has up-regulation 
of the EGFR. ${ }^{34}$ However the clinical benefit of cetuximab appears to be independent of expression of EGFR. ${ }^{35-37}$

Normal/non-mutated KRAS (wild-type) is vital for effect of cetuximab. ${ }^{38}$ Normal expression and mutation status of PTEN and PI3K, are also needed for normal function of the EGFR pathway, as these systems are needed to propagate signals further downstream. ${ }^{39,40}$

Age related changes in the EGF-receptor have been examined in cell and animal studies. These studies suggest that the EGFR system plays a critical role in age-related changed in the colonic mucosa. ${ }^{41}$ Expression of EGFR as well as its activity has been shown to increases with age. ${ }^{42,43}$ However the observations in these animal and in vitro studies have so far not had any clinical relevance.

\section{Pharmacokinetics}

Based on phase I studies, cetuximab was approved with a recommended weekly schedule, and an initial dose of $400 \mathrm{mg} / \mathrm{m}^{2}$ followed by $250 \mathrm{mg} / \mathrm{m}^{2}$ weekly. Peak concentration is reached within approximately 2 hours, with a $T_{1 / 2}$ of approximately 90 hours. ${ }^{44-47}$ The primary elimination of cetuximab is through binding and internalization of the antibody/receptor complex. Secondary elimination after saturation of EGFR, is a slower degradation in the liver and spleen via proteolytic interactions of the $\mathrm{F}_{\mathrm{c}}$ receptors. ${ }^{48}$

Tabernero et al were first to suggest an alternative schedule of cetuximab as they showed that a biweekly regimen (cetuximab $500 \mathrm{mg} / \mathrm{m}^{2}$ every 2 weeks) has a similar pharmacokinetic profile as the approved weekly schedule. ${ }^{49,50}$ A clinical study has demonstrated that the efficacy and toxicity of biweekly cetuximab/irinotecan is similar to results obtained with standard weekly cetuximab/irinotecan. ${ }^{51}$ These results have been confirmed and prospective trials are ongoing. 52

Since the pharmacokinetic profile of cetuximab is independent of liver metabolism, interactions due to polypharmacy are not to be expected. Reduction of dosage of cetuximab in the elderly based on pharmacokinetic data is not advised.

\section{Efficacy and safety of cetuximab in metastatic CRC \\ Second-line and third-line therapy with cetuximab}

Efficacy of cetuximab was initially proven in patients with chemo-resistant mCRC. ${ }^{5,18}$ The promising activity observed in phase I and II studies was first confirmed in the pivotal BOND study where 329 patients with irinotecan-resistent mCRC were randomized to receive either weekly single agent cetuximab (400 mg/m² loading dose IV in 120 minutes, followed by $250 \mathrm{mg} / \mathrm{m}^{2} \mathrm{IV}$ in 60 minutes every week) alone or cetuximab (as above) in combination with irinotecan (administered 60 minutes after cetuximab, resulting in an overall treatment time of more than 3 hours). ${ }^{53}$ The combination significantly increased response rate from $11 \%$ to $23 \%$ and prolonged PFS from 1.5 months to 4.1 months. OS was not significantly prolonged, perhaps due to cross-over and use of cetuximab/irinotecan as salvage therapy at the time of progressive disease (PD) in patients who were randomized to cetuximab. The MABEL study confirmed the results of the BOND study in a large community practice setting in which 1147 patients with irinotecan-refractory mCRC were treated with cetuximab/irinotecan, response rate was $20 \%$ and OS was 9.2 months. ${ }^{54}$

One of the criticisms of the BOND study was the lack of a control group and therefore NCIC-CO.17 was planned and completed. ${ }^{55}$ In this important phase III study, 572 patients pretreated with irinotecan and oxaliplatin were randomized to receive best supportive care (BSC) or cetuximab monotherapy (Table 2a). The primary aim was to confirm an advantage in OS. Compared to BSC, cetuximab was associated with a significant improvement in PFS and OS. The median OS was 6.1 months in the cetuximab group and 4.6 months in the BSC group.

A parallel phase III study $(n=463)$ using the fully human anti-EGFR monoclonal antibody panitumumab (Vectibix ${ }^{\circledR}$ ) confirmed the value of EGFR inhibition in pre-treated patients with mCRC. ${ }^{56}$ Patients received panitumumab or BSC every 2 weeks until PD. Response rate and PFS was significantly improved but OS was not significantly prolonged, perhaps due to cross-over and use of panitumumab as salvage therapy in 172 of 232 patients initially treated with BSC. Panitumumab was approved for monotherapy of refractory mCRC by the US Food and Drug Administration in September 2006 and conditionally approved in patients with tumors harboring wild-type KRAS by the European Medicines Agency (EMEA) in December 2007. ${ }^{6}$ Presently there are no solid data on the combination of panitumumab and chemotherapy. In patients with allergic reactions to cetuximab, re-treatment with cetuximab is possible if patients receive pre-medication, but panitumumab is a good alternative in these patients. ${ }^{57,58}$

The EPIC trial with $1298 \mathrm{mCRC}$ patients resistant to oxaliplatin showed that second-line cetuximab/irinotecan 
Table 2a Selected cetuximab studies in patients with metastatic colorectal cancer

\begin{tabular}{|c|c|c|c|c|c|}
\hline Author, year & Regimen & No of patients & RR (\%) & Median PFS (months) & Median OS (months) \\
\hline \multicolumn{6}{|l|}{ Third-line therapy } \\
\hline \multirow[t]{2}{*}{ Cunningham et al $2004^{53}$} & Cet & III & 11 & 1.5 & 6.9 \\
\hline & Cet + Iri & 218 & $23^{*}$ & $4.1^{*}$ & 8.5 \\
\hline \multirow[t]{2}{*}{ Jonker et al $2007^{55}$} & BSC & 285 & 0 & 1.8 & 4.6 \\
\hline & Cet & 287 & $7^{*}$ & $1.9 *$ & $6.1^{*}$ \\
\hline \multicolumn{6}{|l|}{ Second-line therapy } \\
\hline \multirow[t]{2}{*}{ Sobrero et al $2008^{59}$} & Iri & 650 & 4 & 2.6 & 10.0 \\
\hline & Cet + Iri & 648 & $16 *$ & $4.0^{*}$ & 10.7 \\
\hline \multicolumn{6}{|l|}{ First-line therapy } \\
\hline \multirow[t]{2}{*}{ Van Cutsem 200761 } & FOLFIRI & 598 & 39 & 8.0 & - \\
\hline & FOLFIRI + Cet & 598 & $47^{*}$ & $8.9 *$ & - \\
\hline \multirow[t]{2}{*}{ Bokemeyer et al $2007^{62}$} & FOLFOX & 169 & 36 & 7.2 & - \\
\hline & FOLFOX + Cet & 168 & 46 & 7.2 & - \\
\hline
\end{tabular}

*Significant difference.

Abbreviations: Cet, cetuximab; Iri, irinotecan; BSC, best supportive care.

significantly increased response rate (16\% versus $4 \%)$ and improved PFS (4.0 months versus 2.6 months) over just irinotecan alone. EPIC was designed to study OS, but there was no difference in OS between the two treatment arms. Nearly half of the irinotecan patients were given cetuximab when their cancers progressed and this rescue therapy might explain the lack of significant difference in OS..$^{59}$

Indirectly these data suggest that cetuximab/irinotecan increase response rate to more than $20 \%$, prolong PFS from less than 2 months to more than 4 months and that OS is prolonged from around 5 months to 9 months.

As a result of the BOND study the combination of cetuximab/irinotecan was approved for patients with irinotecan-resistant disease in US and Europe in $2004 .^{5}$

\section{First-line therapy with cetuximab}

Several phase II studies have shown promising activity for chemotherapy-cetuximab combinations as first-line therapy with response rates as high as $80 \%$, high liver resection rates and long OS. ${ }^{18,60}$ Recently the first phase III data (Table 2a) confirmed efficacy of cetuximab in combination with irinotecan or oxaliplatin regimens. ${ }^{61,62}$

In the CRYSTAL study more than 1200 patients with EGFR-expressing mCRC were randomized to FOLFIRI or FOLFIRI + cetuximab. ${ }^{61}$ Response rate (39\% versus $47 \%$ ) and resection rate were higher and PFS was significantly prolonged (8.0 months versus 8.9 months). The higher response rate and longer PFS were also observed in the OPUS study in which 368 patients with $\mathrm{mCRC}$ were randomized to FOLFOX with or without cetuximab. ${ }^{62}$

\section{Predicting efficacy of cetuximab}

High costs of targeted therapies warrant the selection of patients that actually benefit from the therapy. Until recently, the development of skin rash during cetuximab therapy was the most promising predictive factor, but focus has changed towards assessment of tumor tissue. . $^{18,53,63}$

EGFR expression cannot be used to predict efficacy because there is no difference in activity in patients with EGFR positive and EGFR negative tumors. ${ }^{5,35,64-66}$

\section{Mutations in KRAS}

The extracellular EGFR has an impact on stimulating growth in cancer. A number of intracellular downstream regulating molecules including KRAS reinforce this signal. Mutation in KRAS maintains the growth signal even though the extracellular receptor is inhibited. In that way extracellular inhibition is largest in patients with KRAS wild-type (non-mutated), which seems to be the case with approximately $60 \%$ of mCRC patients.

In recent years an increasing number of phase II studies have demonstrated that KRAS mutant status is a predictive marker for lack of efficacy of cetuximab (and panitumumab), as tumor regression in patients with KRAS mutations is very unusual. KRAS mutation status, on the other hand, is not in itself a prognostic marker. ${ }^{67,68}$

In a small study with only $30 \mathrm{mCRC}$ patients, KRAS mutations were strongly associated with lack of response to cetuximab and with a shorter median OS. ${ }^{69}$ These preliminary data were subsequently confirmed in a larger series of 114 patients. ${ }^{38}$ No patient with KRAS mutations obtained response to therapy in contrast to $44 \%$ of patients with KRAS 
wild-type. In addition, patients with a KRAS mutation had a significantly shorter PFS (2.1 months versus 7.4 months) and shorter OS (10.1 months versus 14.3 months). Several comparable retrospective studies have shown similar data. ${ }^{37,70-73}$

In these studies, most of patients received cetuximabirinotecan in combination but some patients also received cetuximab or panitumumab as monotherapy. At the 2008 ASCO meeting, Di Fiori presented data of 281 patients with irinotecan-resistant $\mathrm{mCRC}$ who were treated with cetuximab-irinotecan in several different studies. ${ }^{74}$ KRAS mutations were detected in $35 \%$ of patients. No patient with KRAS mutations obtained regression of the tumor - in contrast to a response rate of $44 \%$ in patients with KRAS wild-type. Median PFS (5.0 months versus 3.0 months) and OS (13.2 months versus 8.0 months) were significantly prolonged in KRAS wild-type patients.

Recent updates of the two large phase III studies comparing BSC and therapy with cetuximab or panitumumab have confirmed that KRAS analysis presently is the best way to identify patients with the lowest chance to benefit from EGFR-inhibition, as only 1 patient among a total of more than 160 patients with KRAS mutations achieved response to EGFR-inhibition. ${ }^{55,56,67,68}$

At the 2008 ASCO GI Cancer meeting, Tabernero et al demonstrated that KRAS status is also associated with efficacy of cetuximab in patients receiving first-line therapy for $\mathrm{mCRC}$. Patients received initially cetuximab monotherapy followed 6 weeks later by a combination of FOLFIRI and cetuximab. ${ }^{75}$ KRAS mutations were detected in $41 \%$ of the tumors. In the first part of the study, patients with KRAS wild-type had a response rate of $27.6 \%$ compared with $0 \%$ for patients with KRAS mutations. In the second part of the study response rate was higher (55\% versus $32 \%)$ and PFS was significantly longer (9.4 months versus 5.6 months) for patients with KRAS wild-type.
In the CRYSTAL study more than 1200 patients were randomized and the authors succeeded in collecting and evaluating KRAS status in tumor tissue from 540 patients who were representative of the total population (Table $2 b$ ). KRAS mutations were detected in 192 patients (35.6\%). In patients with KRAS wild-type, response rate (43\% versus 59\%) and PFS (8.7 months versus 9.9 months) was significantly improved. ${ }^{76}$ In the OPUS study a similar improvement in response rate (37\% versus 61\%) and PFS (7.2 months versus 7.7 months) was presented at the ASCO 2008 meeting. ${ }^{77}$ Survival data in CRYSTAL were presented at ESMO 2008. The improvement in OS (21.0 months versus 24.9 months) showed a non-significant trend in favor of cetuximab, but in patients with a KRAS mutations OS did not differ between the treatment arms (17.5 versus 17.7 months). ${ }^{78}$ The authors concluded that cetuximab added to chemotherapy in patients with KRAS wild-type tumors demonstrated an even more pronounced benefit than that seen in unselected patients and that patients with KRAS mutations seem not to benefit from cetuximab treatment.

Based on data from the CRYSTAL trial and the OPUS trial, the European Commission has extended its cetuximab license to first-line treatment of mCRC patients with KRAS wild-type tumors, in combination with chemotherapy.

KRAS mutation status is a predictive marker for all relevant clinical endpoints in patients with $\mathrm{mCRC}$ receiving treatment with cetuximab and chemotherapy. The efficacy of cetuximab therapy seems confined to patients with KRAS wild-type and data suggest that KRAS status should be analyzed in all patients with mCRC before therapy with cetuximab is commenced.

Ongoing studies will prospectively evaluate efficacy of cetuximab in combination with chemotherapy in patients whose tumors contain KRAS wild-type. In the Nordic 7.5 phase II trial, patients with KRAS wild-type are treated

Table 2b Selected randomized cetuximab studies in patients with KRAS wild-type metastatic colorectal cancer

\begin{tabular}{|c|c|c|c|c|c|}
\hline Author, year & Regimen & No of patients & RR (\%) & Median TTP (months) & Median OS (months) \\
\hline \multicolumn{6}{|l|}{ Third-line therapy } \\
\hline \multirow[t]{2}{*}{ Karapetis et al $2008^{68}$} & BSC & 113 & 0 & 1.9 & 4.8 \\
\hline & Cet & 117 & $11 *$ & $3.8^{*}$ & $9.5 *$ \\
\hline \multicolumn{6}{|l|}{ First-line therapy } \\
\hline \multirow[t]{2}{*}{ Van Cutsem et al $2008^{78}$} & FOLFIRI & 176 & 43 & 8.7 & 21.0 \\
\hline & FOLFIRI + Cet & 172 & $59 *$ & $9.9 *$ & 24.9 \\
\hline \multirow[t]{2}{*}{ Bokemeyer et al $2008^{77}$} & FOLFOX & 73 & 37 & 7.2 & - \\
\hline & FOLFOX + Cet & 61 & $61 *$ & $7.7^{*}$ & - \\
\hline
\end{tabular}


with a combination of Nordic FLOX and biweekly cetuximab ( $500 \mathrm{mg} / \mathrm{m}^{2}$ iv in 60 minutes every second week) for a total of 8 courses ( 4 months) followed by biweekly cetuximab until progression. ${ }^{79}$

\section{Cetuximab in elderly patients}

Data from prospective clinical trials evaluating cetuximab in elderly patients have not been published or presented but planned or un-planned subgroup analysis in patients receiving cetuximab with or without irinotecan is available.

In the BOND study median age was only 59 years and efficacy data have not been correlated to age but elderly patients more than 80 years were included. ${ }^{53}$ In the NCIC-CO.17 study there was no upper age limit, median age was 63 years and patients above 80 years were treated. ${ }^{55}$ In a planned subgroup analysis, no significant differences in the relative benefit (PFS or OS) of cetuximab were seen across subgroups as age (separated by the age of 65 years). In the "panitumumab-study" median age was 62 years and patients above 80 years were also included. ${ }^{56}$ In accordance with NCIC-CO.17, an equivalent relative benefit of cetuximab in terms of PFS was seen in a subgroup defined on age (also separated by the age of 65 years). Furthermore, the significant effect of cetuximab therapy seen in all patients with KRAS wild-type was also retrieved in elderly patients. ${ }^{67}$

A single retrospective study has examined efficacy and safety of cetuximab in elderly patients with mCRC. ${ }^{80}$ Fifty-six patients received cetuximab, most often in combination with irinotecan. The median age was as high as 76 years ( 70 to 84 years) and most patients were pretreated with fluoropyrimidines, irinotecan and oxaliplatin. Response rate was $21 \%$, median PFS was 4.4 months and median OS was 16.0 months.

Our own experience is that efficacy and toxicity are not related to age if therapy is selected according to performance status and co-morbidity. In 74 patients who received biweekly cetuximab/irinotecan ${ }^{51}$, we found no correlation between age and outcome or toxicity; however only 12 patients and 5 patients were older than 70 years and 75 years, respectively. Efficacy data divided according to age (65 years) are shown in Table 3.

Lelli et al have recently published an outcome study of 144 patients receiving weekly cetuximab (usually in combination with irinotecan) for chemo-resistent mCRC. Median PFS and OS were 4.0 months and 11.8 months, respectively. In univariate analysis performance status, weight loss, alkaline phosphatases and skin toxicity were significantly correlated to outcome. ${ }^{81}$ They also divided patients according to age and in accordance with our own outcome study ${ }^{51}$ Lelli et al found similar efficacy in elderly patients $(65+$ years $)$.

\section{Toxicity of cetuximab}

Side effects of cetuximab are related to EGFR expression in normal tissues. In addition, administration of chimeric

Table 3 Sub-group analysis according to age for 74 patients receiving biweekly cetuximab and irinotecan ${ }^{51}$

\begin{tabular}{|c|c|c|}
\hline Characteristic & Biweekly cetlri & \\
\hline Age & $<65$ years & $\geq 65$ years \\
\hline Number & 28 & 46 \\
\hline \multicolumn{3}{|l|}{ WHO performance status } \\
\hline 0 & 20 & 19 \\
\hline 1 & 21 & 8 \\
\hline 2 & 5 & 0 \\
\hline 3 & 0 & 1 \\
\hline Duration of Cetlri, months (range) & $4.7(0.5-12.2)$ & $4.2(0.5-12.8)$ \\
\hline \multicolumn{3}{|l|}{ Response rate } \\
\hline Complete response (CR) & $0(0 \%)$ & $\mathrm{I}(3 \%)$ \\
\hline Partial response (PR) & $14(30 \%)$ & $5(18 \%)$ \\
\hline Stable disease (NC) & $21(46 \%)$ & $17(61 \%)$ \\
\hline Disease control $(\mathrm{CR}+\mathrm{PR}+\mathrm{NC})$ & $35(76 \%)$ & $23(82 \%)$ \\
\hline Progression (PD) & $10(22 \%)$ & $4(14 \%)$ \\
\hline Not evaluable & I (2\%) & I (4\%) \\
\hline PFS, months $(95 \% \mathrm{Cl}), \mathrm{P}=0.2$ & $5.3(3.5-6.7)$ & $5.8(4.6-8.6)$ \\
\hline OS, months $(95 \% \mathrm{Cl}), \mathrm{p}=0.6$ & $8.6(6.3-10.8)$ & $9.1(6.4-11.8)$ \\
\hline
\end{tabular}

Abbreviations: Cetlri, cetuximab and irinotecan; PFS, progression-free survival; OS, overall survival. 
antibodies also may give rise to severe allergic reactions in $1.4 \%-4.5 \% .^{51,53,55,59}$ If patients develop a severe hypersensitivity reaction to cetuximab, panitumumab may be used..$^{58,82,83}$

The most frequent side effect is an acne-like rash primarily located to seborrheic areas. ${ }^{84}$ The skin reactions are fully reversible within a couple of weeks after cessation of therapy. ${ }^{85}$ Several options have been evaluated to ameliorate skin toxicity. In a randomized placebo-controlled study, systemic tetracycline significantly decreased the severity of skin reactions but did not reduce the incidence of rash. ${ }^{86}$ Treatment with oral minocycline and topical tazarotene has also been tested in a randomized trial. Topical tazarotene showed no effect, whereas oral minocycline significantly decreased the number of lesions and itching. ${ }^{87}$

Cetuximab may cause nausea and diarrhea due to effects on the EFGR in the gastro-intestinal tract. ${ }^{53,88}$ EGFR is strongly expressed in the kidneys, particularly in the ascending limb of the loop of Henle where the main part of filtered magnesium is reabsorbed. Blockade of the EGFR in the kidney may trigger hypomagnesemia and in as many as $25 \%$ of patients grade $3-4$ hypomagnesemia has been observed. ${ }^{89,90} \mathrm{~A}$ recent study has shown that the elderly develop hypomagnesemia more rapidly than the younger. ${ }^{91}$ Hypomagnesemia may be corrected by oral supplements or IV infusions; however both modalities can be cumbersome and ineffective. ${ }^{91,92}$ Development of hypomagnesemia may be a predictive marker of outcome..$^{93}$

Less frequently reported side effects are paronychia seen after 2 to 4 months of cetuximab therapy and extensive growth of both eyelashes and eyebrows after long-term treatment with cetuximab. ${ }^{84,85,94}$

There is no evidence that the side-effects of cetuximab, apart from hypomagnesemia, are more severe in elderly patients than in younger patients.

\section{Quality of life}

In an interview based study setting up hypothetical vignettes concerning decisions about treatment, elderly patients did not differ from younger in terms of acceptance of chemotherapy. However, they differ in terms of willingness to trade survival for quality of life. ${ }^{95}$

Assessment of quality of life has been an important part of several cetuximab studies. EPIC and NCIC-CO.17 demonstrated an improved physical function and global health score for patients receiving cetuximab and patients also experienced a significantly longer time before quality of life deteriorated.

\section{Final remarks}

Medical treatment of patients with $\mathrm{mCRC}$ has changed dramatically in the past 5 years, improving survival to 2 years, and there is no evidence that this should not be the case in fit elderly patients.

KRAS status must be analyzed in patients with mCRC before therapy with cetuximab is initiated because efficacy of cetuximab therapy seems confined to patients with KRAS wild-type. In these patients there is strong evidence for adding cetuximab to first-line chemotherapy and for treatment of patients with cetuximab and irinotecan after failure of irinotecan- and oxaliplatin-based therapies. Subgroup analyses of patients included in trials suggest that elderly patients benefits as much from treatment with cetuximab in combination with chemotherapy as younger patients. However in the group of frail elderly patients, studies are needed to make any final conclusions.

\section{Disclosures}

The authors have no conflicts of interest to disclose.

\section{References}

1. Edwards BK, Howe HL, Ries LA, et al. Annual report to the nation on the status of cancer, 1973-1999, featuring implications of age and aging on US cancer burden. Cancer. 2002;94(10):2766-2792.

2. Talarico L, Chen G, Pazdur R. Enrollment of elderly patients in clinical trials for cancer drug registration: a 7-year experience by the US Food and Drug Administration. J Clin Oncol. 2004;22(22):4626-4631.

3. Hutchins LF, Unger JM, Crowley JJ, Coltman CA Jr, Albain KS. Underrepresentation of patients 65 years of age or older in cancer-treatment trials. N Engl J Med. 1999;341(27):2061-2067.

4. Pfeiffer P, Qvortrup C, Eriksen JG. Current role of antibody therapy in patients with metastatic colorectal cancer. Oncogene. 2007;26(25):3661-3678.

5. Nygren P, Sorbye H, Osterlund P, Pfeiffer P. Targeted drugs in metastatic colorectal cancer with special emphasis on guidelines for the use of bevacizumab and cetuximab: an Acta Oncologica expert report. Acta Oncol. 2005;44(3):203-217.

6. Peeters M, Balfour J, Arnold D. Review article: panitumumab - a fully human anti-EGFR monoclonal antibody for treatment of metastatic colorectal cancer. Aliment Pharmacol Ther. 2008;28(3):269-281.

7. Cunningham D, Pyrhonen S, James RD, et al. Randomised trial of irinotecan plus supportive care versus supportive care alone after fluorouracil failure for patients with metastatic colorectal cancer. Lancet. 1998;352(9138):1413-1418.

8. Rougier P, Van CE, Bajetta E, et al. Randomised trial of irinotecan versus fluorouracil by continuous infusion after fluorouracil failure in patients with metastatic colorectal cancer. Lancet. 1998;352(9138): 1407-1412.

9. Meyerhardt JA, Mayer RJ. Systemic therapy for colorectal cancer. NEngl J Med. 2005;352(5):476-487.

10. Grothey A, Sargent D, Goldberg RM, Schmoll HJ. Survival of patients with advanced colorectal cancer improves with the availability of fluorouracil-leucovorin, irinotecan, and oxaliplatin in the course of treatment. J Clin Oncol. 2004;22(7):1209-1214.

11. Tournigand C, Andre T, Achille E, et al. FOLFIRI followed by FOLFOX6 or the reverse sequence in advanced colorectal cancer: a randomized GERCOR study. J Clin Oncol. 2004;22(2):229-237.

12. Glimelius B, Sorbye H, Balteskard L, et al. A randomized phase III multicenter trial comparing irinotecan in combination with the Nordic bolus 5-FU and folinic acid schedule or the bolus/infused de Gramont schedule (Lv5FU2) in patients with metastatic colorectal cancer. Ann Oncol. 2008;19(5):909-914. 
13. Cassidy J, Clarke S, az-Rubio E, et al. Randomized phase III study of capecitabine plus oxaliplatin compared with fluorouracil/folinic acid plus oxaliplatin as first-line therapy for metastatic colorectal cancer. J Clin Oncol. 2008;26(12):2006-2012.

14. Goldberg RM, Sargent DJ, Morton RF, et al. A randomized controlled trial of fluorouracil plus leucovorin, irinotecan, and oxaliplatin combinations in patients with previously untreated metastatic colorectal cancer. J Clin Oncol. 2004;22(1):23-30.

15. Grothey A, Sargent D. Overall survival of patients with advanced colorectal cancer correlates with availability of fluorouracil, irinotecan, and oxaliplatin regardless of whether doublet or single-agent therapy is used first line. J Clin Oncol. 2005;23(36):9441-9442.

16. Koopman M, Antonini NF, Douma J, et al. Sequential versus combination chemotherapy with capecitabine, irinotecan, and oxaliplatin in advanced colorectal cancer (CAIRO): a phase III randomised controlled trial. Lancet. 2007;370(9582):135-142.

17. Seymour MT, Maughan TS, Ledermann JA, et al. Different strategies of sequential and combination chemotherapy for patients with poor prognosis advanced colorectal cancer (MRC FOCUS): a randomised controlled trial. Lancet. 2007;370(9582):143-152.

18. Pfeiffer P, Nielsen D, Yilmaz M, Iversen A, Vejlo C, Jensen BV. Cetuximab and irinotecan as third line therapy in patients with advanced colorectal cancer after failure of irinotecan, oxaliplatin and 5-fluorouracil. Acta Oncol. 2007;46(5):697-6701.

19. Andre T, Tournigand C, Mineur L, et al. Phase II study of an optimized 5-fluorouracil-oxaliplatin strategy (OPTIMOX2) with celecoxib in metastatic colorectal cancer: a GERCOR study. Ann Oncol. 2007; 18(1):77-81.

20. Labianca R, Floriani I, Cortesi E, et al. Alternating versus continuous "FOLFIRI" in advanced colorectal cancer (ACC): A randomized "GISCAD" trial. J Clin Oncol. (ASCO Meeting Abstracts) 2006;24(18 suppl):3505.

21. Maindrault-Goebel F, Tournigand C, Andre T, et al. Oxaliplatin reintroduction in patients previously treated with leucovorin, fluorouracil and oxaliplatin for metastatic colorectal cancer. Ann Oncol. 2004 August;15(8):1210-4.

22. Perez-Staub N, Chibaudel B, Figer A, et al. Who can benefit from chemotherapy holidays after first-line therapy for advanced colorectal cancer? A GERCOR study. J Clin Oncol. (Meeting Abstracts) 2008;26(15 suppl):4037.

23. Popescu RA, Norman A, Ross PJ, Parikh B, Cunningham D. Adjuvant or palliative chemotherapy for colorectal cancer in patients 70 years or older. J Clin Oncol. 1999;17(8):2412-2418.

24. Feliu J, Escudero P, Llosa F, et al. Capecitabine as first-line treatment for patients older than 70 years with metastatic colorectal cancer: an oncopaz cooperative group study. J Clin Oncol. 2005;23(13):3104-3411.

25. Daniele B, Rosati G, Tambaro R, et al. First-line chemotherapy with fluorouracil and folinic acid for advanced colorectal cancer in elderly patients: a phase II study. J Clin Gastroenterol. 2003;36(3):228-233.

26. Chiara S, Nobile MT, Vincenti M, et al. Advanced colorectal cancer in the elderly: results of consecutive trials with 5-fluorouracil-based chemotherapy. Cancer Chemother Pharmacol. 1998;42(4):336-40.

27. Sargent DJ, Goldberg RM, Jacobson SD, et al. A pooled analysis of adjuvant chemotherapy for resected colon cancer in elderly patients. N Engl J Med. 2001;345(15):1091-1097.

28. Goldberg RM, Tabah-Fisch I, Bleiberg H, et al. Pooled analysis of safety and efficacy of oxaliplatin plus fluorouracil/leucovorin administered bimonthly in elderly patients with colorectal cancer. J Clin Oncol. 2006;24(25):4085-4091.

29. Folprecht G, Seymour MT, Saltz L, et al. Irinotecan/fluorouracil combination in first-line therapy of older and younger patients with metastatic colorectal cancer: combined analysis of 2,691 patients in randomized controlled trials. J Clin Oncol. 2008;26(9):1443-1451.

30. Pentheroudakis G, Fountzilas G, Kalofonos HP, et al. Palliative chemotherapy in elderly patients with common metastatic malignancies: A Hellenic Cooperative Oncology Group registry analysis of management, outcome and clinical benefit predictors. Crit Rev Oncol Hematol. 2008;66(3):237-247.
31. Sanoff HK, Bleiberg H, Goldberg RM. Managing older patients with colorectal cancer. J Clin Oncol. 2007;25(14):1891-1897.

32. McKibbin T, Frei CR, Greene RE, Kwan P, Simon J, Koeller JM. Disparities in the use of chemotherapy and monoclonal antibody therapy for elderly advanced colorectal cancer patients in the community oncology setting. Oncologist. 2008;13(8):876-885.

33. Carpenter G, Cohen S. Epidermal growth factor. J Biol Chem. 1990;265(14):7709-7712.

34. Goldstein NS, Armin M. Epidermal growth factor receptor immunohistochemical reactivity in patients with American Joint Committee on Cancer Stage IV colon adenocarcinoma: implications for a standardized scoring system. Cancer. 2001;92(5):1331-1346.

35. Chung KY, Shia J, Kemeny NE, et al. Cetuximab shows activity in colorectal cancer patients with tumors that do not express the epidermal growth factor receptor by immunohistochemistry. J Clin Oncol. 2005;23(9):1803-1810.

36. Moroni M, Veronese S, Benvenuti S, et al. Gene copy number for epidermal growth factor receptor (EGFR) and clinical response to antiEGFR treatment in colorectal cancer: a cohort study. Lancet Oncol. 2005;6(5):279-286.

37. Khambata-Ford S, Garrett CR, Meropol NJ, et al. Expression of epiregulin and amphiregulin and K-ras mutation status predict disease control in metastatic colorectal cancer patients treated with cetuximab. J Clin Oncol. 2007;25(22):3230-3237.

38. Lievre A, Bachet JB, Boige V, et al. KRAS mutations as an independent prognostic factor in patients with advanced colorectal cancer treated with cetuximab. J Clin Oncol. 2008;26(3):374-379.

39. Jhawer M, Goel S, Wilson AJ, et al. PIK3CA mutation/PTEN expression status predicts response of colon cancer cells to the epidermal growth factor receptor inhibitor cetuximab. Cancer Res. 2008;68(6):1953-1961.

40. Frattini M, Saletti P, Romagnani E, et al. PTEN loss of expression predicts cetuximab efficacy in metastatic colorectal cancer patients. Br J Cancer. 2007;97(8):1139-1145.

41. Xiao ZQ, Moragoda L, Jaszewski R, Hatfield JA, Fligiel SE, Majumdar AP. Aging is associated with increased proliferation and decreased apoptosis in the colonic mucosa. Mech Ageing Dev. 2001;122(15):1849-1864.

42. Tureaud J, Sarkar FH, Fligiel SE, et al. Increased expression of EGFR in gastric mucosa of aged rats. Am J Physiol. 1997;273(2 Pt 1): G389-G398.

43. Xiao ZQ, Majumdar AP. Increased in vitro activation of EGFR by membrane-bound TGF-alpha from gastric and colonic mucosa of aged rats. Am J Physiol Gastrointest Liver Physiol. 2001;281(1):G111-G116.

44. Fracasso PM, Burris H, III, Arquette MA, et al. A phase 1 escalating single-dose and weekly fixed-dose study of cetuximab: pharmacokinetic and pharmacodynamic rationale for dosing. Clin Cancer Res. 2007 February 1;13(3):986-93.

45. Dirks NL, Nolting A, Kovar A, Meibohm B. Population pharmacokinetics of cetuximab in patients with squamous cell carcinoma of the head and neck. J Clin Pharmacol. 2008;48(3):267-278.

46. Baselga J, Pfister D, Cooper MR, et al. Phase I studies of anti-epidermal growth factor receptor chimeric antibody $\mathrm{C} 225$ alone and in combination with cisplatin. J Clin Oncol. 2000;18(4):904-914.

47. Tan AR, Moore DF, Hidalgo M, et al. Pharmacokinetics of cetuximab after administration of escalating single dosing and weekly fixed dosing in patients with solid tumors. Clin Cancer Res. 2006;12(21):6517-6522.

48. Tabrizi MA, Tseng CM, Roskos LK. Elimination mechanisms of therapeutic monoclonal antibodies. Drug Discov Today. 2006; 11(1-2):81-88.

49. Tabernero J, Pfeiffer P, Cervantes A. Administration of cetuximab every 2 weeks in the treatment of metastatic colorectal cancer: an effective, more convenient alternative to weekly administration? Oncologist. 2008;13(2):113-119.

50. Tabernero J, Cervantes A, Martinelli E, et al. Optimal dose of cetuximab (C) given every 2 weeks (q2w): A phase I pharmacokinetic (PK) and pharmacodynamic (PD) study of weekly (q1w) and q2w schedules in patients (pts) with metastatic colorectal cancer (mCRC). J Clin Oncol. (Meeting Abstracts) 2006;24(18 suppl):3085. 
51. Pfeiffer P, Nielsen D, Bjerregaard J, Qvortrup C, Yilmaz M, Jensen B. Biweekly cetuximab and irinotecan as third-line therapy in patients with advanced colorectal cancer after failure to irinotecan, oxaliplatin and 5-fluorouracil. Ann Oncol. 2008;19(6):1141-1145.

52. Martin-Martorell P, Rosello S, Rodriguez-Braun E, Chirivella I, Bosch A, Cervantes A. Biweekly cetuximab and irinotecan in advanced colorectal cancer patients progressing after at least one previous line of chemotherapy: results of a phase II single institution trial. Br J Cancer. 2008;99(3): 455-458.

53. Cunningham D, Humblet $Y$, Siena $S$, et al. Cetuximab monotherapy and cetuximab plus irinotecan in irinotecan-refractory metastatic colorectal cancer. $N$ Engl J Med. 2004;351(4):337-345.

54. Wilke H, Glynne-Jones R, Thaler J, et al. MABEL-A large multinational study of cetuximab plus irinotecan in irinotecan resistant metastatic colorectal cancer. J Clin Oncol. (Meeting Abstracts) 2006;24(18 suppl):3549.

55. Jonker DJ, O'Callaghan CJ, Karapetis CS, et al. Cetuximab for the treatment of colorectal cancer. N Engl J Med. 2007;357(20):2040-2048.

56. Van CE, Peeters M, Siena S, et al. Open-label phase III trial of panitumumab plus best supportive care compared with best supportive care alone in patients with chemotherapy-refractory metastatic colorectal cancer. J Clin Oncol. 2007;25(13):1658-1664.

57. Nielsen DL, Pfeiffer P, Jensen BV. Re-treatment with cetuximab in patients with severe hypersensitivity reactions to cetuximab. Two case reports. Acta Oncologica. 2006;45(8):1137-1138.

58 Cartwright TH, Genther R. Successful administration of panitumumab alone after severe infusion reaction to cetuximab in a patient with metastatic colorectal cancer. Clin Colorectal Cancer. 2008;7(3):202-203.

59. Sobrero AF, Maurel J, Fehrenbacher L, et al. EPIC: phase III trial of cetuximab plus irinotecan after fluoropyrimidine and oxaliplatin failure in patients with metastatic colorectal cancer. J Clin Oncol. 2008;26(14):2311-2319.

60. Macarulla T, Ramos FJ, Elez E, Capdevila J, Peralta S, Tabernero J. Update on novel strategies to optimize cetuximab therapy in patients with metastatic colorectal cancer. Clin Colorectal Cancer. 2008;7(5):300-308.

61. Van Cutsem E, Nowacki M, Lang I, et al. Randomized phase III study of irinotecan and 5-FU/FA with or without cetuximab in the firstline treatment of patients with metastatic colorectal cancer (mCRC): The CRYSTAL trial. $J$ Clin Oncol. (ASCO Meeting Abstracts) 2007;25(18 suppl):4000.

62. Bokemeyer C, Bondarenko I, Makhson A, et al. Cetuximab plus 5-FU/FA/ oxaliplatin (FOLFOX-4) versus FOLFOX-4 in the first-line treatment of metastatic colorectal cancer (mCRC): OPUS, a randomized phase II study. J Clin Oncol. (Meeting Abstracts) 2007;25(18 suppl):4035.

63. Lenz HJ, Van CE, Khambata-Ford S, et al. Multicenter phase II and translational study of cetuximab in metastatic colorectal carcinoma refractory to irinotecan, oxaliplatin, and fluoropyrimidines. $J$ Clin Oncol. 2006;24(30):4914-4921.

64. Hebbar M, Wacrenier A, Desauw C, et al. Lack of usefulness of epidermal growth factor receptor expression determination for cetuximab therapy in patients with colorectal cancer. Anticancer Drugs. 2006;17(7):855-857.

65. Meropol NJ. Epidermal growth factor receptor inhibitors in colorectal cancer: it's time to get back on target. J Clin Oncol. 2005;23(9): 1791-1793.

66. Hecht J, Mitchell E, Baranda J, et al. Panitumumab antitumor activity in patients (pts) with metastatic colorectal cancer (mCRC) expressing low $(1 \%-9 \%)$ or negative $(<1 \%)$ levels of epidermal growth factor receptor (EGFr). J Clin Oncol. (Meeting Abstracts) 2006;24(18 suppl):3547.

67. Amado RG, Wolf M, Peeters M, et al. Wild-Type KRAS is required for panitumumab efficacy in patients with metastatic colorectal cancer. J Clin Oncol. 2008;26(10):1626-1634.

68. Karapetis CS, Khambata-Ford S, Jonker DJ, et al. K-ras mutations and benefit from cetuximab in advanced colorectal cancer. $N$ Engl J Med. 2008;359(17):1757-1765

69. Lievre A, Bachet JB, Le CD, et al. KRAS mutation status is predictive of response to cetuximab therapy in colorectal cancer. Cancer Res. 2006;66(8):3992-3995.
70. Benvenuti S, Sartore-Bianchi A, Di NF, et al. Oncogenic activation of the RAS/RAF signaling pathway impairs the response of metastatic colorectal cancers to anti-epidermal growth factor receptor antibody therapies. Cancer Res. 2007;67(6):2643-2648.

71. De Roock W, Piessevaux H, De SJ, et al. KRAS wild-type state predicts survival and is associated to early radiological response in metastatic colorectal cancer treated with cetuximab. Ann Oncol. 2008;19(3):508-515.

72. Finocchiaro G, Cappuzzo F, Janne PA, et al. EGFR, HER2 and Kras as predictive factors for cetuximab sensitivity in colorectal cancer. $J$ Clin Oncol. (Meeting Abstracts) 2007;25(18 suppl):4021.

73. Di Fiore F, Blanchard F, Charbonnier F, et al. Clinical relevance of KRAS mutation detection in metastatic colorectal cancer treated by Cetuximab plus chemotherapy. Br J Cancer. 2007;96(8):1166-1169.

74. Di Fiore F, Van Cutsem E, Laurent-Puig P, et al. Role of KRAS mutation in predicting response, progression-free survival, and overall survival in irinotecan-refractory patients treated with cetuximab plus irinotecan for a metastatic colorectal cancer: Analysis of 281 individual data from published series. J Clin Oncol. (Meeting Abstracts) 2008;26(15 suppl):4035.

75 Tabernero J, Cervantes A, Ciardiello F. Correlation of efficacy to KRAS status (wt vs. mut) in patients (pts) with metastatic colorectal cancer $(\mathrm{mCRC})$, treated with weekly ( $\mathrm{q} 1 \mathrm{w})$ and $\mathrm{q} 2 \mathrm{w}$ schedules of cetuximab combined with FOLFIRI. ASCO GI 2008, Abstract number:435.

76. Van Cutsem E, Lang I, D'haens G, et al. KRAS status and efficacy in the first-line treatment of patients with metastatic colorectal cancer (mCRC) treated with FOLFIRI with or without cetuximab: The CRYSTAL experience. J Clin Oncol. (Meeting Abstracts) 2008;26(15 suppl):2.

77. Bokemeyer C, Bondarenko I, Hartmann JT, et al. KRAS status and efficacy of first-line treatment of patients with metastatic colorectal cancer (mCRC) with FOLFOX with or without cetuximab: The OPUS experience. J Clin Oncol. (Meeting Abstracts) 2008;26(15 suppl):4000.

78. Van Cutsem E, Lang I, D'haens G. Kras status and efficacy in the CRYSTAL Study: 1st-line treatment of patients with metastatic colorectal cancer (mCRC) receiving FOLFIRI with or without cetuximab. Ann Oncol. 2008;19 (Supp 8):710.

79. Sorbye H, Glimelius B, Berglund A, et al. Multicenter phase II study of Nordic fluorouracil and folinic acid bolus schedule combined with oxaliplatin as first-line treatment of metastatic colorectal cancer. J Clin Oncol. 2004;22(1):31-38.

80. Bouchahda M, Macarulla T, Spano JP, et al. Cetuximab efficacy and safety in a retrospective cohort of elderly patients with heavily pretreated metastatic colorectal cancer. Crit Rev Oncol Hematol. 2008;67(3):255-262.

81. Lelli G, Cataldo S, Carandina I, et al. The role of cetuximab in pre-treated refractory patients with metastatic colorectal cancer: outcome study in clinical practice. J Chemother. 2008;20(3):374-379.

82. Saif MW, Peccerillo J, Potter V. Successful re-challenge with panitumumab in patients who developed hypersensitivity reactions to cetuximab: report of three cases and review of literature. Cancer Chemother Pharmacol. 2008 Sep 10. [Epub ahead of print] PMID: 18781300.

83. Heun J, Holen K. Treatment with panitumumab after a severe infusion reaction to cetuximab in a patient with metastatic colorectal cancer: a case report. Clin Colorectal Cancer. 2007;6(7):529-531.

84. Saltz LB, Meropol NJ, Loehrer PJ Sr, Needle MN, Kopit J, Mayer RJ. Phase II trial of cetuximab in patients with refractory colorectal cancer that expresses the epidermal growth factor receptor. J Clin Oncol. 2004;22(7):1201-1208.

85. Robert C, Soria JC, Spatz A, et al. Cutaneous side-effects of kinase inhibitors and blocking antibodies. Lancet Oncol. 2005;6(7):491-500.

86. Jatoi A, Rowland K, Sloan JA, et al. Tetracycline to prevent epidermal growth factor receptor inhibitor-induced skin rashes: results of a placebo-controlled trial from the North Central Cancer Treatment Group (N03CB). Cancer. 2008;113(4):847-853.

87. Scope A, Agero AL, Dusza SW, et al. Randomized double-blind trial of prophylactic oral minocycline and topical tazarotene for cetuximabassociated acne-like eruption. J Clin Oncol. 2007;25(34):5390-5396. 
88. Vincenzi B, Santini D, Rabitti C, et al. Cetuximab and irinotecan as third-line therapy in advanced colorectal cancer patients: a single centre phase II trial. Br J Cancer. 2006;94(6):792-797.

89. Schrag D, Chung KY, Flombaum C, Saltz L. Cetuximab therapy and symptomatic hypomagnesemia. J Natl Cancer Inst. 2005;97(16): 1221-1224.

90. Carson EJ, Novak AM, Stella PJ. Hypomagnesemia in patients with stage IV colorectal cancer treated with cetuximab as a single agent. J Clin Oncol. (Meeting Abstracts) 2005;23(16 suppl):3655.

91. Tejpar S, Piessevaux H, Claes K, et al. Magnesium wasting associated with epidermal-growth-factor receptor-targeting antibodies in colorectal cancer: a prospective study. Lancet Oncol. 2007;8(5):387-394.

92. Fakih MG, Wilding G, Lombardo J. Cetuximab-induced hypomagnesemia in patients with colorectal cancer. Clin Colorectal Cancer. 2006;6(2):152-156.

93. Vincenzi B, Santini D, Galluzzo S, et al. Early magnesium reduction in advanced colorectal cancer patients treated with cetuximab plus irinotecan as predictive factor of efficacy and outcome. Clin Cancer Res. 2008;14(13):4219-4224.

94. Bouche O, Brixi-Benmansour H, Bertin A, Perceau G, Lagarde S. Trichomegaly of the eyelashes following treatment with cetuximab. Ann Oncol. 2005;16(10):1711-1712.
95. Yellen SB, Cella DF, Leslie WT. Age and clinical decision making in oncology patients. J Natl Cancer Inst. 1994;86(23):1766-1770.

96. Saltz LB, Cox JV, Blanke C, et al. Irinotecan plus fluorouracil and leucovorin for metastatic colorectal cancer. Irinotecan Study Group. N Engl J Med. 2000;343(13):905-914.

97. Douillard JY, Cunningham D, Roth AD, et al. Irinotecan combined with fluorouracil compared with fluorouracil alone as first-line treatment for metastatic colorectal cancer: a multicentre randomised trial. Lancet. 2000;355(9209):1041-1047.

98. Kohne CH, Van CE, Wils J, et al. Phase III study of weekly high-dose infusional fluorouracil plus folinic acid with or without irinotecan in patients with metastatic colorectal cancer: European Organisation for Research and Treatment of Cancer Gastrointestinal Group Study 40986. J Clin Oncol. 2005;23(22):4856-4865.

99. De GA, Figer A, Seymour M, et al. Leucovorin and fluorouracil with or without oxaliplatin as first-line treatment in advanced colorectal cancer. J Clin Oncol. 2000;18(16):2938-2947.

100. Giacchetti S, Perpoint B, Zidani R, et al. Phase III multicenter randomized trial of oxaliplatin added to chronomodulated fluorouracil-leucovorin as first-line treatment of metastatic colorectal cancer. J Clin Oncol. 2000;18(1):136-147. 
